\title{
Combined transpapillary drainage and endoscopic ultrasound-guided hepatico- duodenostomy after failed manipulation under cholangioscopy guidance
}

For unresectable malignant hilar biliary obstructions, drainage of $\geq 50 \%$ of the liver volume is recommended [1]. Endoscopic transpapillary biliary drainage is the current standard treatment for malignant hilar biliary obstruction. Cholangioscopy-assisted guidewire placement is a useful method for biliary drainage, which can be used even in patients without contrast-filled images [2]

In recent years, the use of endoscopic ultrasound-guided biliary drainage (EUSBD) for malignant hilar biliary obstruction has increasingly been reported. However, it is technically challenging, especially in patients with right intrahepatic bile duct
(IHBD) obstruction. Herein, we report the use of combined conventional transpapillary drainage and EUS-guided hepaticoduodenostomy (EUS-HDS) in a patient with malignant hilar biliary obstruction after failed manipulation under cholangioscopy guidance.
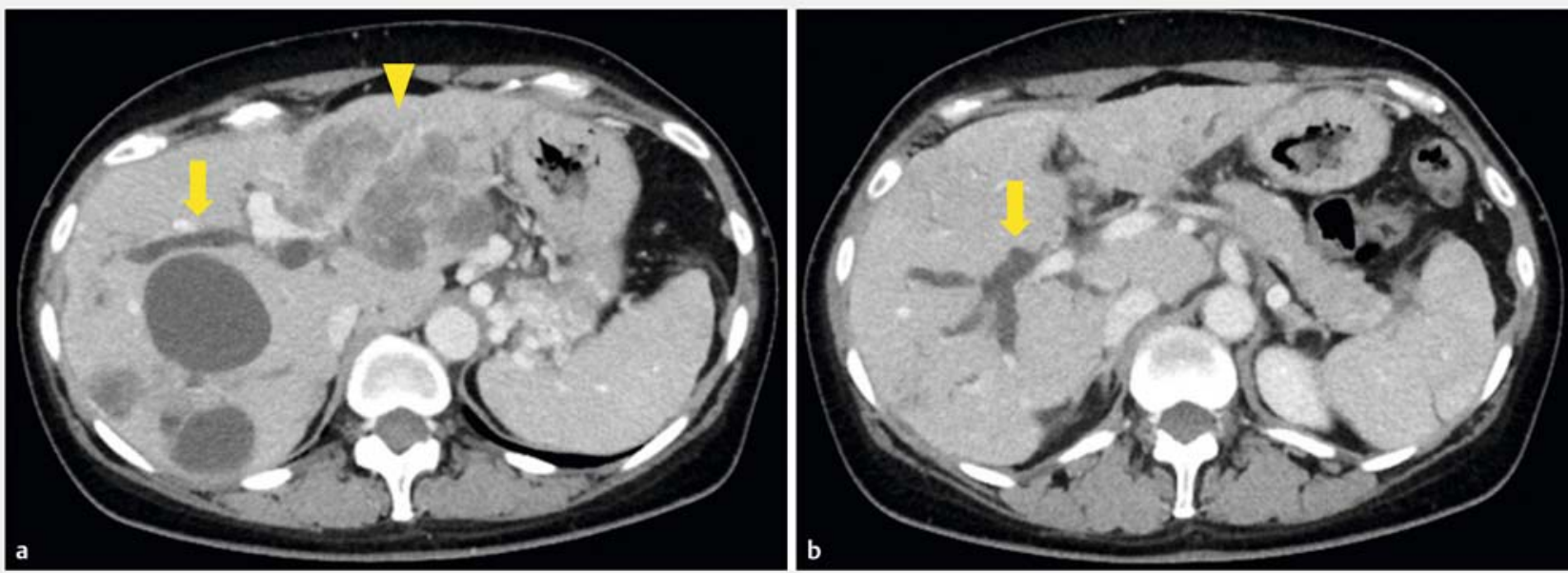

- Fig. 1 Radiographic images showing: a the left lobe of the liver affected by metastasis (yellow triangle); a, $\mathbf{b}$ the surrounding lymph node metastases causing malignant hilar biliary obstruction (yellow arrow).
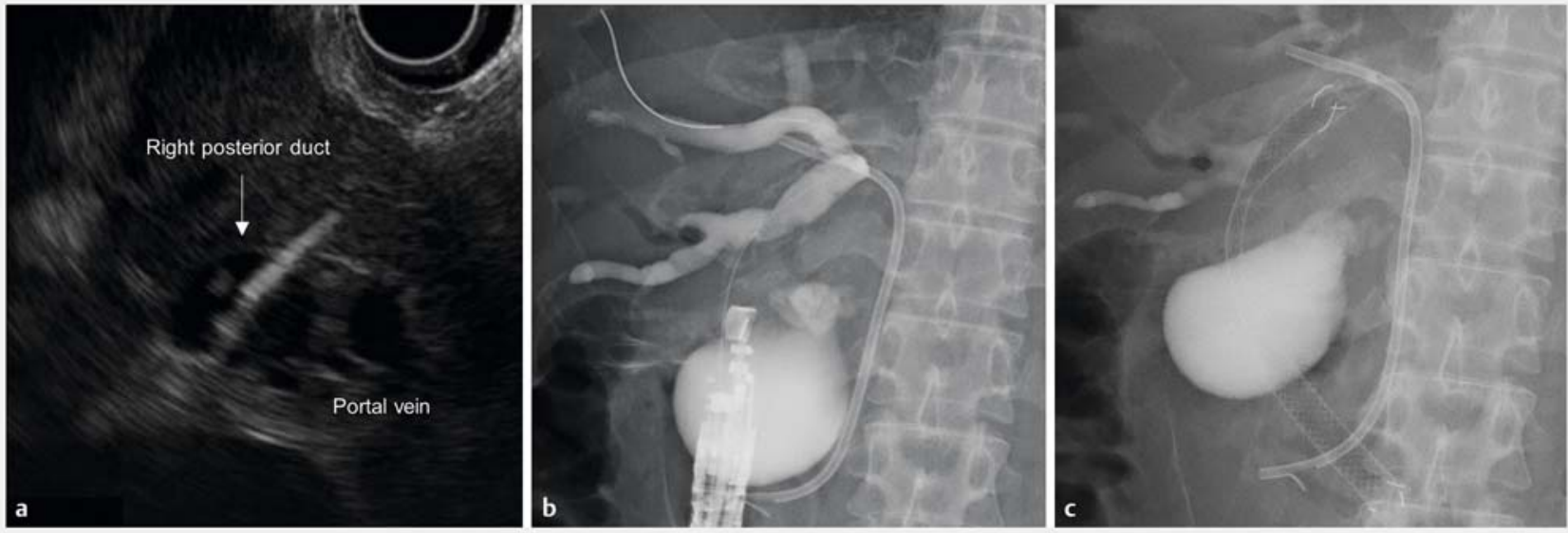

- Fig. 2 Images during endoscopic ultrasound (EUS)-guided right hepaticoduodenostomy showing: a on EUS image, the right posterior biliary duct being punctured with a $19 \mathrm{G}$ needle ; b on fluoroscopy, a 0.025-inch guidewire introduced into the posterior intrahepatic bile duct; c a successfully placed partially covered metal stent in the bile duct. 


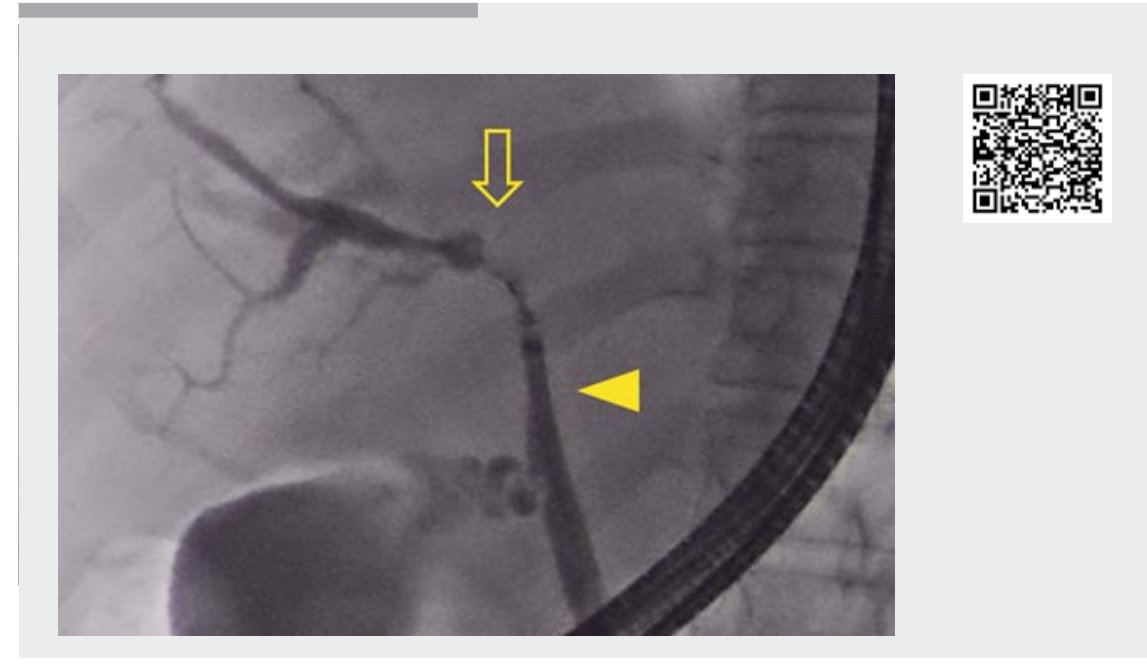

Video 1 A single session of combined conventional transpapillary biliary drainage and endoscopic ultrasound-guided hepaticoduodenostomy (EUS-HDS) in a patient with malignant hilar biliary obstruction (MHBO) after failed manipulation under cholangioscopy guidance.

A 46-year-old woman was diagnosed with malignant hilar biliary obstruction caused by unresectable pancreatic cancer, with metastasis affecting the left lobe of the liver ( $\triangleright$ Fig. 1). For effective and precise biliary decompression, drainage of both the right anterior and posterior IHBDs was required; however, we were only able to insert the guidewire into the anterior IHBD. Because the cholangiogram showed complete obstruction, intraductal cholangioscopic guidance (SpyClass DS system; Boston Scientific Corp., Natick, Massachusetts, USA) was used to access the posterior IHBD. Despite direct visualization of the bile duct, we could not access the posterior IHBD. Therefore, we subsequently used a linear echoendoscope and punctured the posterior IHBD through the duodenal wall, using a 19G needle (EZ Shot 3 Plus; Olympus Medical Systems, Tokyo, Japan). A 0.025-inch guidewire (VisiGlide 2; Olympus Medical Systems) was then placed, which allowed balloon dilation to $4 \mathrm{~mm}$, and finally the successfully placement into the bile duct of a partially covered metal stent (Niti-S stent, $8 \times 120 \mathrm{~mm}$; Taewoong Medical, Seoul, Korea) to create an EUS-HDS (॰ Fig. 2; $\triangleright$ Video 1 ).

For EUS-BD of the right-side of the liver, two main techniques have been described: direct puncture of the rightside of the liver from the bulbus (EUSHDS), as first reported by Park et al. in
2013 [3], and a bridge technique that allows drainage of the right-side of the liver from the left via hepaticogastrostomy (the bridge technique) [4]. In this case, the latter technique could not be applied because of the left-sided liver metastasis; the former was feasible, but only 13 further cases of the technique have been reported [5]. In cases such as this, where we were unable to obtain a cholangiogram using a contrast agent, EUS-BD might be preferable because of its rapidity and cost-effectiveness. We would emphasize that, for technically demanding biliary drainage, there are alternatives to conventional transpapillary biliary drainage, including EUS-BD.

\section{Endoscopy_UCTN_Code_TTT_1AS_2AD}

\section{Competing interests}

The authors declare that they have no conflict of interest.

\section{The authors}

\section{Yasuki Hori, Kazuki Hayashi, Itaru Naitoh,} Michihiro Yoshida, Makoto Natsume, Hidenori Sahashi, Hiromi Kataoka Department of Gastroenterology and Metabolism, Nagoya City University Graduate School of Medical Sciences, Nagoya, Japan
Corresponding author

\section{Kazuki Hayashi, MD, PhD}

Department of Gastroenterology and Metabolism, Nagoya City University Graduate School of Medical Sciences, 1 Kawasumi, Mizuho-cho, Mizuho-ku, Nagoya 467-8601, Japan

Fax: +81-52-8520952

khayashi@med.nagoya-cu.ac.jp

References

[1] Vienne A, Hobeika E, Gouya $\mathrm{H}$ et al. Prediction of drainage effectiveness during endoscopic stenting of malignant hilar strictures: the role of liver volume assessment. Gastrointest Endosc 2010; 72: 728-735

[2] Yoshida M, Kato A, Hayashi K et al. Novel technique for intraductal cholangioscopyassisted biliary drainage with over-the-wire microcatheter manipulation. Endoscopy 2019; 51: E398-E399

[3] Park S], Choi JH, Park DH et al. Expanding indication: EUS-guided hepaticoduodenostomy for isolated right intrahepatic duct obstruction (with video). Gastrointest Endosc 2013; 78: 374-380

[4] Ogura T, Sano T, Onda S et al. Endoscopic ultrasound-guided biliary drainage for right hepatic bile duct obstruction: novel technical tips. Endoscopy 2015; 47: 72-75

[5] Caillol F, Rouy M, Pesenti C et al. Drainage of the right liver using EUS guidance. Endosc Ultrasound 2019; 8: S50-S56

\section{Bibliography}

Endoscopy 2021; 53: E153-E154

DOI 10.1055/a-1216-1254

ISSN 0013-726X

published online 20.8.2020

(c) 2020. Thieme. All rights reserved.

Georg Thieme Verlag KG, Rüdigerstraße 14, 70469 Stuttgart, Germany

\section{ENDOSCOPY E-VIDEOS \\ https://eref.thieme.de/e-videos}

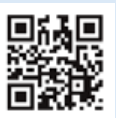

Endoscopy E-Videos is a free access online section, reporting on interesting cases and new techniques in gastroenterological endoscopy. All papers include a high quality video and all contributions are freely accessible online.

This section has its own submission website at https://mc.manuscriptcentral.com/e-videos 